\title{
Audit of peripheral neuromuscular stimulators at the hospitals staffed by the Department of Anaesthesia and Perioperative Medicine at the University of Cape Town
}

\author{
AT Joubert, ${ }^{1}$ O Porrill, ${ }^{1}$ J Paed ${ }^{2}$ \\ ${ }^{1}$ Department of Anaesthesia, University of Cape Town, South Africa \\ ${ }^{2}$ Department of Electrical Engineering, University of Cape Town, South Africa \\ Corresponding author, email: andries.thomas.joubert@gmail.com
}

Background: Inadequate monitoring of neuromuscular blockade (NMB) may result in worse patient outcomes, including airway compromise and postoperative pulmonary complications. Therefore, NMB monitor availability is a minimum requirement for perioperative care according to the South African Society of Anaesthesiologists' (SASA) 2018 guidelines. The authors performed an audit of peripheral nerve stimulators (PNS) functionality and availability at their institution.

Methods: The PNSs were attached to an electrical circuit with a skin equivalent resistance. The resultant current impulses generated using Train-of-Four (TOF) mode and Double Burst Stimulation (DBS) mode were recorded with a voltage scope meter. PNS availability was assessed in theatre and recovery areas.

Results: Of the 65 PNS units assessed, 39 units were dysfunctional and 26 units fully functional. The most frequent fault found (30 units) related to faulty or absent PNS electrode cables. Eight functional PNS units with TOF ratio display capability were found. The working PNSs showed good inter-device peak voltage measurement correlation. Of the 59 areas identified where PNSs should be easily available, only 37 areas met the PNS availability criteria suggested in the SASA guidelines.

Conclusion: This audit highlighted the need for more new generation PNSs with TOF-ratio-display-ability to align the institution with recommendations from SASA standards and the anaesthetic literature. It also highlighted the accuracy and consistency of delivered current bursts by the working PNS devices.

Keywords: peripheral nerve stimulator, peripheral neuromuscular monitor, postoperative residual curarization, postoperative neuromuscular blockade, residual paralysis, postoperative neuromuscular function

\section{Introduction}

\section{History and practice of NMB monitoring}

The first muscle relaxant, d-tubocurarine, was introduced into anaesthetic practice in 1942 by Harold Griffith. ${ }^{1}$ As early as 1954, it was noted that patients who had received d-tubocurarine had a six-fold higher mortality rate than patients who had not received the drug. ${ }^{2}$ Inadequate reversal of skeletal muscle paralysis at the end of anaesthesia has been described as postoperative residual curarization (PORC). In 1958, the use of a nerve stimulator to monitor the action of muscle relaxants was suggested, ${ }^{3}$ but for many years thereafter only clinical parameters were used to infer the absence of clinically significant PORC in the recovery area, including: Head lift more than 5 seconds, tongue protrusion, vital capacity $>15 \mathrm{ml} / \mathrm{kg}^{4}$ and hand grip strength. ${ }^{5}$

By 1971 it was suggested that the level of PORC could be assessed by using the train-of-four twitch technique (TOF). ${ }^{6}$ A ratio of $>0.7$ was considered to indicate adequate recovery of muscle strength before endotracheal tube removal, given that it correlated well with the clinical parameters mentioned above. This standard was used for many years thereafter. In 1997,7 a significant post-operative pulmonary complication (POPC) rate was reported if the train-of-four value was $<0.7$ in the immediate postoperative period after pancuronium administration.
Around the same time it was demonstrated ${ }^{8}$ (by TOF-testing the adductor pollicis muscles of a small sample of volunteers) that pharyngeal tone approaches normal values when the concurrent TOF demonstrates a ratio of $>0.9$. This cast doubt on the validity of the previously held threshold being adequate for endotracheal tube removal. Subsequently a TOF ratio of $>0.9$ became the accepted standard to achieve before endotracheal tube removal.

Another concern is related to the inability to detect fade by clinical examination during TOF delivery. Diagnosing PORC by tactile or visual assessment of the peripheral nerve stimulation response is sub-optimal. Even experienced observers cannot feel fade if the TOF ratio is $>0.4 .^{9,10}$ Mechanomyography, electromyography and acceleromyography are alternative measurement techniques that provide more objective assessments of PORC. ${ }^{11}$

Recent studies have shown that the TOF ratio is slightly overestimated when it is determined using acceleromyography. Thus it is questioned whether a TOF ratio of 1.0 should rather be aimed for when acceleromyography is used, in order to ensure a TOF ratio $>0.9 . .^{12,13}$

\section{Clinical significance}

It is well described in the literature that PORC leads to increased postoperative complications, including: 
- Airway and breathing compromise

- An unpleasant patient experience ${ }^{14,15}$

- Decreased theatre efficiency

In the South African context two studies reported incidences of $29 \%$ and $43 \%$ respectively for PORC with TOF $<0.9 .{ }^{16,17}$ Elsewhere a meta-analysis in 2007 inferred that PORC is common, with an estimated TOF $<0.9$ incidence rate of about $41 \%$, in patients who received intermediate acting NMBs. ${ }^{18}$

\section{Airway and breathing compromise:}

- A five times higher rate of aspiration (laryngeal penetration) was described in a study of partially paralysed healthy volunteers given an infusion of atracurium with concomitant monitoring of the TOF ratio and video radiography of the larynx. ${ }^{19}$

- PORC is associated with an increased risk of POPC, including respiratory failure, pulmonary oedema, tracheal reintubation and pneumonia. Data from a retrospective analysis of hospital records of 48499 patients showed an association between higher doses of neuromuscular blocking drugs and POPC. ${ }^{20}$

- More episodes of upper airway obstruction during transport to the post anaesthesia care unit (PACU) were observed in patients managed with conventional qualitative TOF assessment compared to similar patients managed with acceleromyographic quantitative TOF monitoring. ${ }^{21}$

- Post-operative hypoxaemia22

- In this randomised prospective placebo controlled trial of 114 patients, failure to reverse minimal PORC was associated with hypoxaemia in the PACU, occurring about twice as often in the no-neostigmine placebo group (29 occurrences) vs. the neostigmine group (16 occurrences).

- Critical respiratory events in the PACU 23

- In this study $0.8 \%$ of patients developed critical respiratory events (61 of 7459 patients), most commonly hypoxaemia and upper airway obstruction. The TOF ratios, mean and ( \pm standard deviation), in the critical respiratory event group were $0.62( \pm 0.2)$.

\section{An unpleasant experience:}

- In one study, 149 patients arriving in PACU were divided into two groups based on a measured TOF ratio of $<0.9$ and $\geq 0.9$ respectively. The 48 patients who fell in the TOF ratio $<0.9$ group were found to have, after arrival in PACU:

- A feeling of general muscle weakness in $73 \%$ at 60 minutes

- Inability to swallow in $29 \%$ at 20 minutes

- Inability to speak normally in $33 \%$ of patients at 40 minutes

- Inability to cough normally in $27 \%$ at 40 minutes

- Blurry vision in $23 \%$ and diplopia in $10 \%$ at 60 minutes.

It is no surprise that some of these symptoms associated with PORC are reported as unpleasant. ${ }^{24}$

\section{Decreased perioperative efficiency}

Longer times to discharge from the PACU has been described for patients with PORC. ${ }^{25}$ In this study of 246 consecutive PACU patient admissions, the average time in PACU was 243 minutes for patients with adequate recovery compared to 323 minutes for patients who had a TOF ratio of $<0.9$.

Slower postoperative weaning from ventilation and longer times to endotracheal tube removal have been associated with lower TOF ratios after cardiac surgery. ${ }^{15}$

\section{Why do some anaesthetists monitor NMB inadequately?}

At the authors' institution, the perceived lack of availability of PNSs could contribute to inadequate monitoring and management of neuromuscular blockade. This encouraged the authors to perform an audit of PNSs.

Worldwide it is described that many anaesthetists are still not monitoring the level of neuromuscular blockade with a quantitative modality such as the TOF ratio. Hypotheses as to why that may be include ${ }^{26}$ : heuristics, confirmation bias, production pressure to optimise turnaround time and complacency bred from past "successes".

\section{What should anaesthetists be doing?}

In South Africa the following recommendations are made with regards to neuromuscular blockade monitoring and reversal ${ }^{27}$ (South African Society of Anaesthesiologists [SASA] 2018):

- A peripheral nerve stimulator to monitor neuromuscular function is considered an essential item needed as a minimum requirement for the safe conduct of anaesthesia in any hospital.

- Peripheral nerve stimulators should be immediately available in theatre and in the recovery room.

- Patients should have recovered from neuromuscular blockade prior to handover to staff from the theatre recovery room, and thus also prior to discharge from the anaesthetist's care.

It is described in the literature that PORC cannot be assessed adequately without an objective measure of neuromuscular function. 910 Taken in conjunction with the SASA recommendations above, it is the authors' opinion that neuromuscular function should be assessed using a PNS device with TOF ratio display capabilities.

\section{Methodology}

\section{Overview}

- The study was designed as an audit of PNS availability and function with the aim to compare our PNS equipment availability to the recommended standard. More than $90 \%$ of data collection was done between 2 January 2018 and 2 February 2018, and the last area was assessed on 15 May 2018.

- Study location: 
This audit was performed at the hospitals staffed by the department of Anaesthesia and Perioperative Medicine at the University of Cape Town, which included the following locations: Groote Schuur Hospital, Mowbray Maternity Hospital, Red Cross War Memorial Children's Hospital, New Somerset Hospital, Maitland Cottage Hospital and Valkenberg Hospital (hereafter referred to as "the authors' institution".)

- Research procedures and data collection methods:

The number of PNSs available in the theatre and recovery areas of the institution was documented. These findings were judged against the 2012 SASA practice guidelines. A unique identification number was assigned to each PNS unit. Thereafter, the functionality of these units was tested by confirming the presence of the electrical current bursts delivered during the TOF and Double Burst Stimulation (DBS) modes. In order to perform these measurements, the PNSs were connected to a skin-equivalent-resistance circuit built by the co-author Mr Pead (Figure 1).

We opted to use a $2.47 \mathrm{kOhm}$ resistor for this, given that between 1 and $4.5 \mathrm{kOhm}$ is described in test manuals. The voltage produced by the electrical current bursts set at $60 \mathrm{~mA}$ were measured over the resistor. Thereafter a graphic display of the voltage/time wave loop of the TOF and DBS current bursts were recorded in numerical (Excel ${ }^{\oplus}$ ) and digital photographic (PNG file) format using a Keysight InfiniiVision EDUX1002G Digital Storage Oscilloscope. The PNSs were classified as either functional or faulty depending on the results of this test, applying Ohm's law. Data were recorded and stored using an Excel spreadsheet.

\section{PNS availability}

PNS units are often shared between operating theatres to save costs. Therefore, it is important to define adequate availability. For the purpose of this study, importance was placed on having a "designated specific location" for the device. If the device was kept in a specifically labelled location just outside theatre, it would be labelled as adequately available. On the other hand, if a unit was shared between theatres but was not kept in a designated location it was deemed "not easily available".

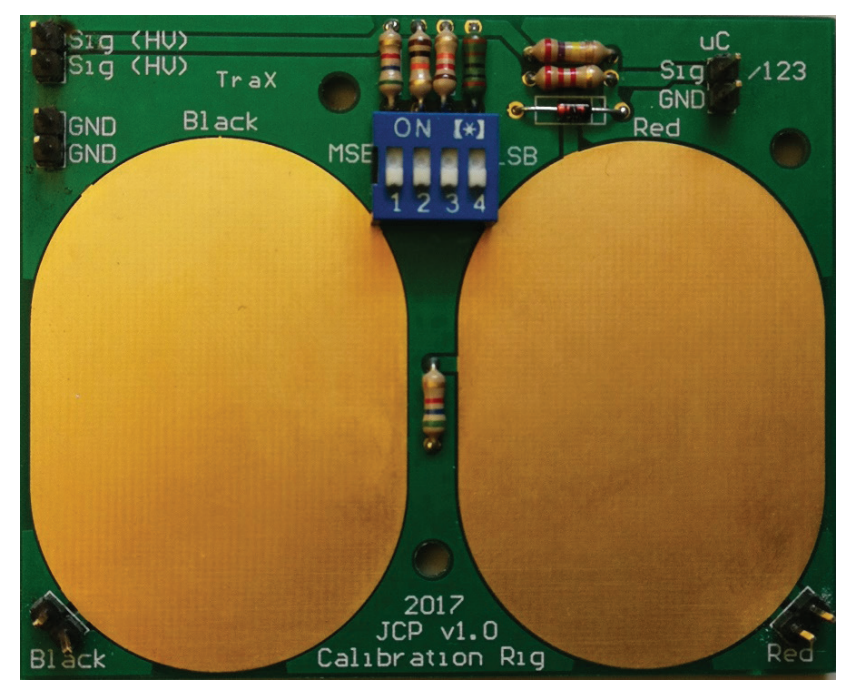

Figure 1: Latest version of resistance circuit for PNS testing

\section{Statistics}

The nerve stimulators were classified as functional or not, and the nature of the faults described. The areas assessed for PNS availability were classified into three groups with PNS either "physically present", "easily available" or "not easily available."

Given the striking repeatability of the PNS recordings between devices, it was opted to use descriptive statistical analysis of this data to demonstrate and emphasise this finding using the mean and standard deviation, once it was confirmed that the data was normally distributed.

\section{Results}

In total 59 areas where a peripheral stimulator should be easily available (PNS-zones) were identified. These areas consisted of 48 operating theatre or procedural areas (e.g. Catheterisation Laboratory, Electroconvulsive Therapy Suite) and 11 recovery areas.

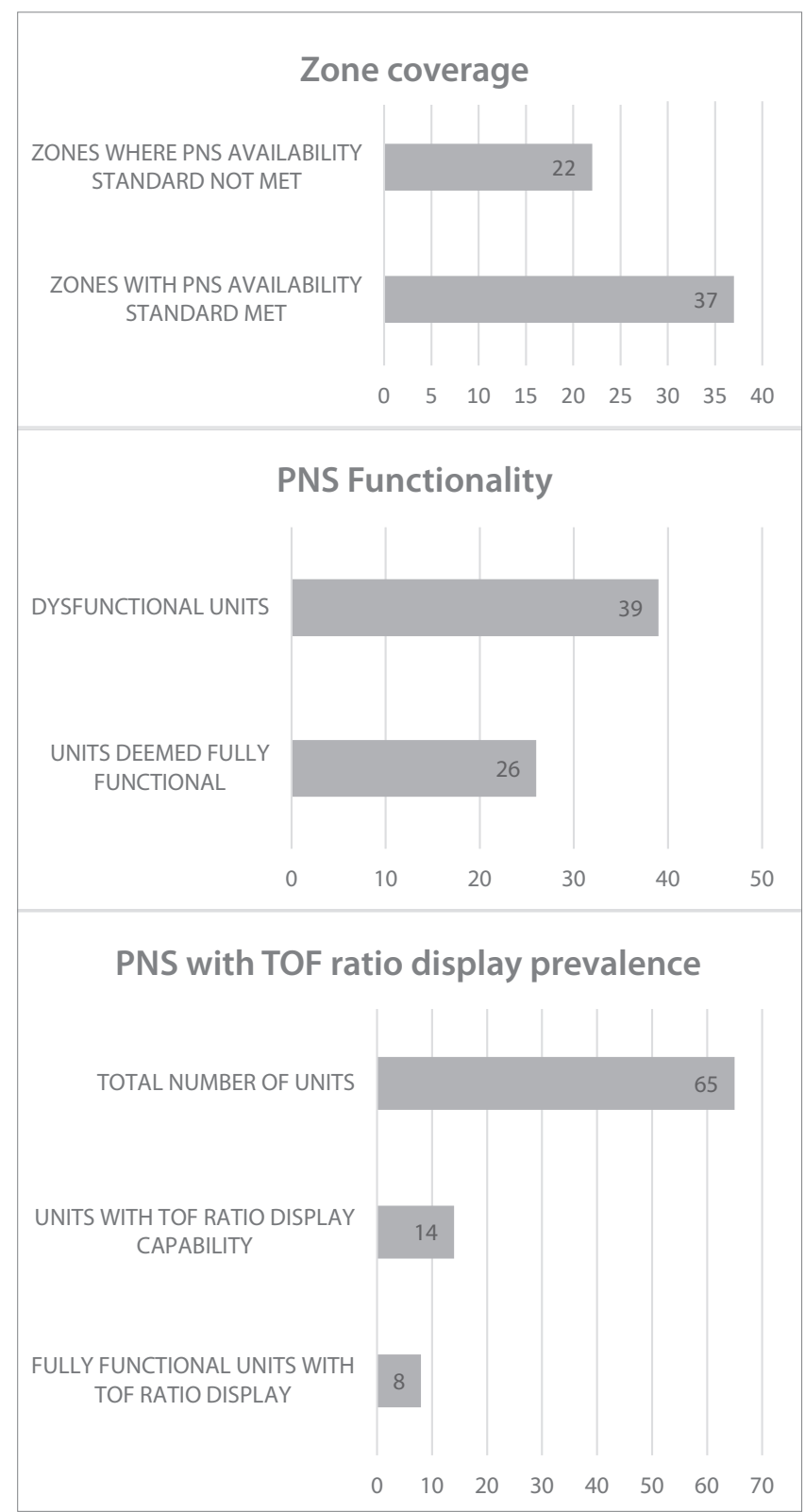

Figure 2: Summary of audit results 


\section{No. of PNS units counted}

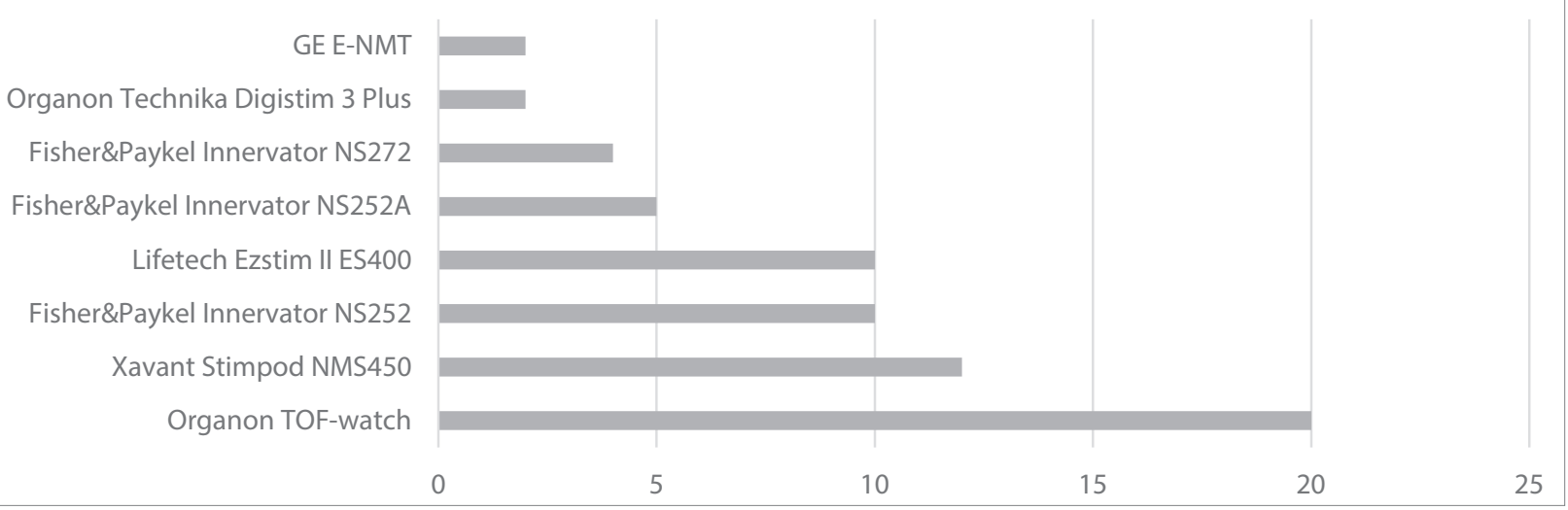

Figure 3: PNS makes and models encountered

\section{Number of units affected:}

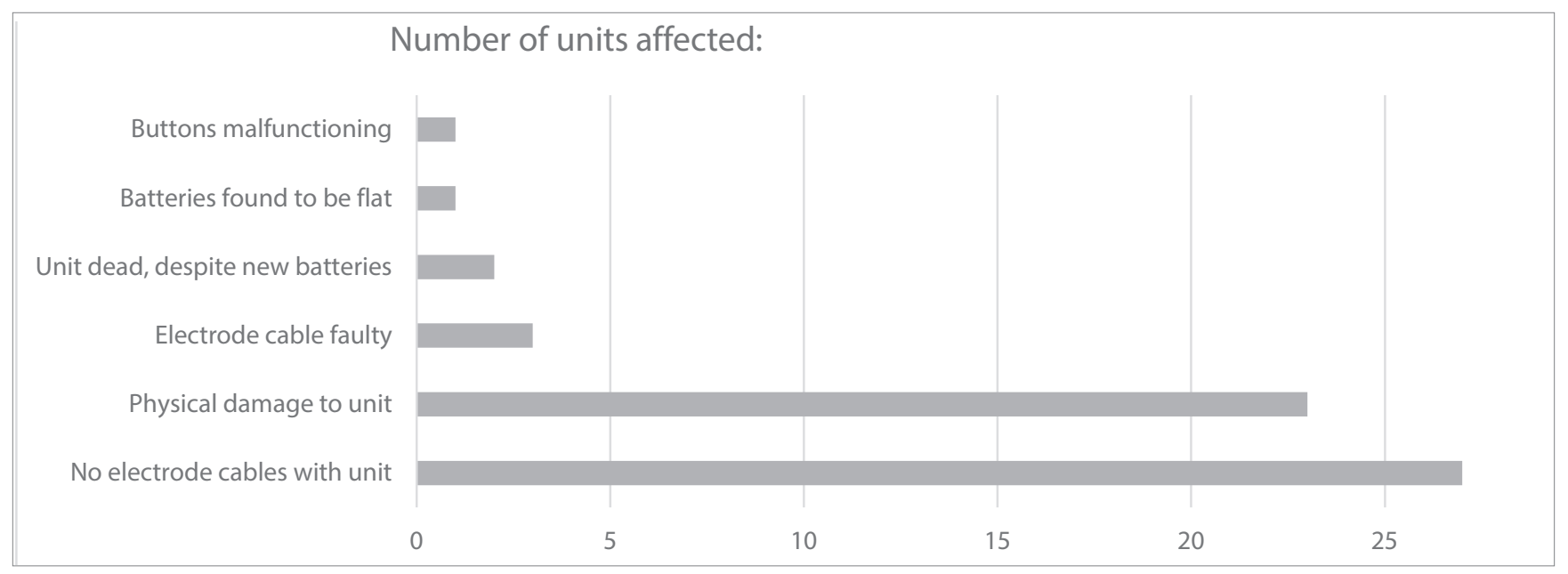

Figure 4: Faults found with PNS units

Of these areas, 37 PNS-zones were deemed to meet the PNS availability criteria suggested in the SASA guidelines. When looking at the data in more detail, one would see that a PNS unit was found to be physically present in 21 areas, easily available in 19 areas and not easily available in 19 areas. Three areas were found with dysfunctional PNSs, resulting in them being classified as available but not meeting the overall availability standard.

TOF-ratio display capability was only represented in 14 of the units. Of these 14 units only 8 were fully functional (Figure 2). 65 PNS units were tested for functionality. The different makes and models are depicted in the chart above (Figure 3). Of these units, 39 were deemed to be dysfunctional and the remaining 26 units were deemed fully functional.

The faults found with the units were as follows:

- Absence of electrode cables was found in 27 units. These cables were either lost during clinical use or discarded by technicians when found to be malfunctioning. Given that many of the units were no longer manufactured or supported by their manufacturers, replacement cables could not be acquired. Only 3 units were found to have faulty cables, but it may well be the most frequent malfunction found on the PNSs, given that a large number of the "missing" cables may reflect original cable malfunction.
- The other malfunctions found are indicated in Figure 4.

The overall consistency of the delivered voltage spikes is noteworthy. In total 61 units were able to give scope meter recordings, although this was often performed with a set of "Ioan cables" where the units' original cables were damaged or missing. This translated into 244 peak values for TOF voltage peak measurements. An assessment was made of the mean and standard deviation of the peak values measured with the scope meter. The skewness and kurtosis suggested normal distribution of the data and there was no sign change from baseline readings with paired t-tests.

\section{Discussion}

The ability to monitor neuromuscular blockade perioperatively is essential given the known adverse effects of PORC ${ }^{19-24}$ and the inability to reliably detect the presence of PORC through clinical parameters alone.9,10 The SASA 2018 practice guidelines also lists peripheral nerve stimulators as an "essential monitor considered to be a minimum requirement for the safe conduct of anaesthesia." ${ }^{27}$

This audit revealed that there were not enough functional PNSs available at the institution. There were 8 functioning TOF-R capable peripheral nerve stimulators available to cover 59 areas. 
If we add the handheld devices without TOF-Ratio capability, 37 areas out of the 59 (63\%) are covered. This confirmed the authors' pre-audit clinical suspicion that there was a shortage of these monitors at the institution.

It can be inferred that it is unlikely that peripheral neuromuscular blockade monitoring is performed to the current accepted standard of a "TOF-Ratio of $>0.9$ " if we have only 8 such devices available to cover 59 PNS zones at our institution.

The clinical significance of the shortage of PNS units varies depending on the actual need for PNMB monitoring in the area concerned. Despite this, the SASA practice guidelines do not make concessions for areas where PNSs are less likely to be used and describe this as an essential monitor that needs to be immediately available. Thus, a lower likelihood of needing the device should not be used as an excuse for inadequate coverage.

The logistics of tracking a PNS unit's location is very important in situations where nerve stimulators have to be shared between locations. In areas where the nerve stimulator may be used in more than 2 locations, immediate availability of the device is lost once it is in use. In order to facilitate locating the device the unit has to be stored in a specific named "base" location, and the whereabouts of the device must be documented once it is removed. This could be done with the use of a simple notebook kept at the "base location", or by online systems such as a "devicelocation-Whatsapp-group" shared between users of the device.

From a technical point of view the working PNSs were found to be very consistent in their delivered current bursts. This information is useful when troubleshooting a PNS unit. Looking at our data set there would be a high likelihood of the electrode cable being the problem rather that the rest of the electronic hardware.

Another possible relevant factor that was not explored in this study is the likelihood of skin resistance contributing to measurement variance. Skin resistance may vary with skin thickness, moisture content and temporal association with prior electrical stimulation. In addition, it is well-known that skin impedance decreases when exposed to higher intensity currents. $^{28}$

There are problems with the older PNS units, which comprise the majority of units in the institution:

- The inability of these devices to exclude incomplete return of neuromuscular function by modern standards make them all but obsolete. However, some value remains in the ability to demonstrate very deep NMB levels where fade is detectable clinically and to demonstrate where TOF counts $<4$ are present.

- The lack of manufacturer support for many of the older PNS units in use at our institution shortens the foreseeable lifespan of the remaining functional PNS fleet. Some of these PNS models are no longer manufactured. Most of these units will thus become unusable at some point when they break down and need maintenance or repairs.

\section{Implications for our region resulting from the audit findings}

- The performance of this audit helped catalyse the acquisition of further modern PNS units at our institution. At the conclusion of this study a further $8 \mathrm{E}-\mathrm{NMT}$ units have already been delivered to GSH hospital and the plan is to continue increasing our inventory for the future.

- Procurement of a modern scope meter with the ability to store and download test data in a downloadable format should be considered for the clinical engineering departments of the hospitals where PNSs are used. This equipment will facilitate routine maintenance and enable future audit projects of electronic devices.

- Hospitals needs to continue acquiring more TOF-R capable units to align themselves with measurement standards described in the literature.

- An awareness and educational campaign needs to be launched within the departments of anaesthesia to educate and motivate anaesthetists to:

- Utilise appropriate PNMB monitoring, and

- Advocate for the provision of adequate numbers of PNS devices.

- The cost and availability of repairs and cable replacements should be factored into the decision when acquiring more PNS units. The anaesthetic machine mounted PNS unit retails for about 3 times the price of the handheld device. It is hypothesised that the machine mounted units may last longer due to:

- Higher quality electrode cabling

- Less handling of the machine mounted units, resulting in

- A smaller chance of being dropped and broken

- Less winding up / coiling of the electrode cables which may prolong cable life.

- The cost-benefit ratio of the current options (E-NMT vs. Xavant Stimpod NMS450) should be further audited prospectively while in use to help guide future acquisitions of these devices.

- There may be a potential market for small cost-effective skinequivalent resistance test-circuit devices that can give a binary confirmation of functionality for a PNS unit delivering TOF and DBS.

\section{Summary}

- Patients should have their neuromuscular function monitored perioperatively during and after the administration of neuromuscular blocking agents until full return of neuromuscular function.

- The practical "standard of care" for neuromuscular function monitoring is the use of acceleromyography in conjunction with device software that will express a train-of-four ratio. ${ }^{29}$

- This audit highlighted the need at the authors' institution for more new generation PNSs with TOF-ratio-display-ability to align ourselves with the recommendations from the anaesthetic literature. 
- It also highlighted the need for an improved sharing system in those areas were PNS: clinical area ratio is $<1$.

- This research also highlighted the accuracy and consistency of delivered current bursts by the working PNS devices.

- There are opportunities for further research in evaluating the contribution of skin related factors on the conduction of current bursts with PNSs.

\section{Ethical approval}

Ethics approval was obtained from the University of Cape Town, Faculty of Health Sciences Human Research Ethics Committee, HREC REF: 771/2017

\section{References}

1. Gillies DMM, Wynands JE. Obituary Dr. Harold Griffith 1894-1985. Can Anaesth Soc J. 1985;32:570-4. 10.1007/bf03010816: 10.1007/bf03010816

2. Beecher HK, Todd DP. A Study of the Deaths Associated with Anesthesia and Surgery: Based on a Study of 599,548 Anesthesias in Ten Institutions 1948-1952, Inclusive. Ann Surg. 1954;140:2-34.

3. Christie TH, Churchill-Davidson HC. The St. Thomas's Hospital nerve stimulator in the diagnosis of prolonged apnoea. Lancet (London, England). 1958;1:776.

4. Brand JB, Cullen DJ, Wilson NE, Ali HH. Spontaneous recovery from nondepolarizing neuromuscular blockade: correlation between clinical and evoked responses. Anesth Analg 1977;56:55-8.

5. Russell WJ, Serle DG. Hand grip force as an assessment of recovery from neuromuscular block. J Clin Monit. 1987;3:87-9.

6. Ali HH, Utting JE, Gray TC. Quantitative assessment of residual antidepolarizing block (Part II). BJA: Br J Anaesth. 1971;43:478-85. 10.1093/bja/43.5.478: 10.1093/ bja/43.5.478

7. Berg H, Viby-Mogensen J, Roed J, et al. Residual neuromuscular block is a risk factor for postoperative pulmonary complications A prospective, randomised, and blinded study of postoperative pulmonary complications after atracurium, vecuronium and pancuronium. Acta Anaesthesiolscand. 1997;41:1095-103. 10.1111/j.1399-6576.1997.tb04851.x: 10.1111/j.1399-6576.1997.tb04851.x

8. Eriksson LI, Sundman E, Olsson R, et al. Functional assessment of the pharynx at rest and during swallowing in partially paralyzed humans: simultaneous videomanometry and mechanomyography of awake human volunteers. Anesthesiology. 1997;87:1035-43.

9. Viby-Mogensen J, Jensen NH, Engbaek J, Ording H, Skovgaard LT, ChraemmerJørgensen B. Tactile and Visual Evaluation of the Response to Train-of-four Nerve Stimulation. Anesthesiology. 1985;63:440-2.

10. Saddler JM, Bevan JC, Donati F, Bevan DR, Pinto SR. Comparison of Double-burst and Train-of-four Stimulation to Assess Neuromuscular Blockade in Children. Anesthesiology. 1990;73:401-3.

11. Viby-Mogenson J. Postoperative residual curarization and evidence-based medicine. Br J Anaesth. 2000;84:301-3.

12. Capron F, Alla F, Hottier C, Meistelman C, Fuchs-Buder T. Can Acceleromyography Detect Low Levels of Residual Paralysis? A Probability Approach to Detect a Mechanomyographic Train-of-four Ratio of 0.9. Anesthesiology. 2004;100:111924. 10.1097/00000542-200405000-00013: 10.1097/00000542-200405000-00013

13. Kopman AF, Klewicka MM, Neuman GG. The relationship between acceleromyographic train-of-four fade and single twitch depression. Anesthesiology. 2002 96:583-7.

14. Murphy GS, Szokol JW, Avram MJ, et al. Postoperative residual neuromuscular blockade is associated with impaired clinical recovery. Anesth Analg. 2013:117:133-41. 10.1213/ANE.0b013e3182742e75: 10.1213/ ANE.0b013e3182742e75

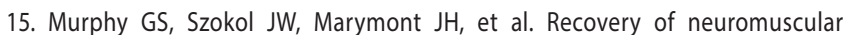
function after cardiac surgery: pancuronium versus rocuronium. Anesth Analg. 2003;96:1301-7.

16. Invernizzi JRR, Gopalan PD. Postoperative neuromuscular function following non-depolarising muscle blockade in patients at Inkosi Albert Luthuli Central Hospital, Durban. Southern African Journal of Anaesthesia and Analgesia. 2016;22:121-4. 10.1080/22201181.2016.1201293: 10.1080/22201181.2016.1201293

17. Nell WT, Stevenson N, Ridgard T, Westhuizen FWVd, Diedericks J, Joubert G. Post-operative neuromuscular function of patients receiving non-depolarising muscle relaxants at Universitas Hospital, Bloemfontein, South Africa: research. Southern African Journal of Anaesthesia and Analgesia. 2004;10:6-8.
18. Naguib M, Kopman AF, Ensor JE. Neuromuscular monitoring and postoperative residual curarisation: a meta-analysis. Br J Anaesth. 2007;98:302-16. 10.1093/bja/ ael386: 10.1093/bja/ael386

19. Sundman E, Witt H, Olsson R, Ekberg O, Kuylenstierna R, Eriksson LI. The Incidence and Mechanisms of Pharyngeal and Upper Esophageal Dysfunction in Partially Paralyzed HumansPharyngeal Videoradiography and Simultaneous Manometry after Atracurium. Anesthesiology:: 2000; 92: 977-84.

20. McLean DJ, Diaz-Gil D, Farhan HN, Ladha KS, Kurth T, Eikermann M Dose-dependent Association between Intermediate-acting Neuromuscularblocking Agents and Postoperative Respiratory Complications. Anesthesiology. 2015;122:1201-13. 10.1097/aln.0000000000000674: 10.1097/ aln.0000000000000674

21. Murphy GS, Szokol JW, Marymont JH, et al. Intraoperative acceleromyographic monitoring reduces the risk of residual blockade and adverse respiratory events in the postanesthesia care unit. Anesthesiology. 2008;109:389-98.

22. Sauer M, Stahn A, Soltesz S, Noeldge-Schomburg G, Mencke T. The influence of residual neuromuscular block on the incidence of critical respiratory events. A randomised, prospective, placebo-controlled trial. Eur J Anaesthesiol. 2011;28:842-8. 10.1097/EJA.0b013e328345cd11: 10.1097/ EJA.0b013e328345cd11

23. Murphy GS, Szokol JW, Marymont JH, Greenberg SB, Avram MJ, Vender JS. Residual neuromuscular blockade and critical respiratory events in the postanesthesia care unit. Anesth Analg. 2008;107:130-7. 10.1213/ ane.0b013e31816d1268: 10.1213/ane.0b013e31816d1268

24. Kopman AF, Yee PS, Neuman GG. Relationship of the train-of-four fade ratio to clinical signs and symptoms of residual paralysis in awake volunteers. Anesthesiology. 1997;86:765-71.

25. Butterly A, Bittner EA, George E, Sandberg WS, Eikermann M, Schmidt U. Postoperative residual curarization from intermediate-acting neuromuscular blocking agents delays recovery room discharge. Br J Anaesth. 2010;105:304-9 10.1093/bja/aeq157: 10.1093/bja/aeq157

26. Brull SJ, Prielipp RC. Reversal of neuromuscular blockade: "identification friend or foe". Anesthesiology. 2015;122:1183-5. 10.1097/aln.0000000000000675: 10.1097/ aln.0000000000000675

27. Kluyts $H$, Sing U, Farina $Z$, et al. South African Society of Anaesthesiologists Practice Guidelines 2018 Revision. [Accessed 0908 2018] 2018.

28. Stephens W. The current-voltage relationship in human skin. Med Electron Biol Eng. 1963;1:389-99.

29. Capron F, Fortier LP, Racine S, Donati F. Tactile fade detection with hand or wrist stimulation using train-of-four, double-burst stimulation, 50-hertz tetanus, 100-hertz tetanus, and acceleromyography. Anesth Analg. 2006;102:1578-84 10.1213/01.ane.0000204288.24395.38: 10.1213/01.ane.0000204288.24395.38

\section{Glossary of terms used:}

DBS - Double Burst Stimulation

NMB - Neuromuscular blockade

PACU - Post anaesthesia care unit

PNS - Peripheral nerve stimulators

PNS-zones - Areas where peripheral stimulator availability is needed according to South African Society for Anaesthesiologists' practice guidelines

PNM - Peripheral neuromuscular monitors (a synonym for PNS)

PORC - Postoperative residual curarization

POPC - Postoperative pulmonary complications

RCWMCH - Red Cross War Memorial Children's Hospital

RNMB - Residual neuromuscular blockade, a synonym for PORC

SASA - South African Society of Anaesthesiologists

TOF - Train-of-four 